\title{
Circulation de la parole et des imaginaires. Sur les pas de Nasreddin Hodja
}

\section{Nadine Decourt}

\section{(2) OpenEdition \\ 12 Journals}

Édition électronique

URL : http://journals.openedition.org/pa/191

DOI : 10.4000/pa.191

ISSN : 2273-0362

Éditeur

Université Lumière Lyon 2

Édition imprimée

Date de publication : 15 janvier 2009

Pagination : 81-91

ISBN : 978-2-912868-62-6

ISSN : 1634-7706

\section{Référence électronique}

Nadine Decourt, «Circulation de la parole et des imaginaires. Sur les pas de Nasreddin Hodja »,

Parcours anthropologiques [En ligne], 7| 2009, mis en ligne le 02 juillet 2013, consulté le 19 avril 2019.

URL : http://journals.openedition.org/pa/191 ; DOI : 10.4000/pa.191 


\title{
Circulation de la parole et des imaginaires Sur les pas de Nasreddin Hodja
}

\author{
NAdine Decourt
}

Il est ici un personnage dont je voudrais valoriser l'émergence et le rayonnement dans le paysage culturel français pour en avoir été le témoin oculaire et interactif. Même sa silhouette nous devient familière, sur les couvertures de livres pour la jeunesse, plus ou moins inspirées de miniatures du XVII ${ }^{e}$ siècle. Il a pour nom Nasreddin Hodja et nous vient principalement de Turquie, monté sur son âne, barbe blanche au vent aussi ronde que sa bedaine ou que les plis de son turban. Le voici sur toutes les scènes du conte en son renouveau multiculturel. S'il est toujours au programme dans les écoles en Turquie, omniprésent sur les présentoirs de brochures pour touristes et depuis longtemps traduit, il a commencé sa vie littéraire côté France en immigré clandestin, à la faveur des opérations interculturelles innovantes des années 1980. Il était plus facile alors de mettre en confiance des élèves et leurs parents, avec de courts récits facétieux venus de chez eux qu'avec les grands contes merveilleux de la parole de nuit. De plus ces historiettes procèdent par cycle, chaîne et associations sans fin de motifs à plaisanterie : le personnage paraît inépuisable et donne à voir toutes les facettes de la vie.

Le suivre dans quelques-unes de ses tribulations nous aidera à mieux comprendre la circulation de la parole et des imaginaires qui s'opère aujourd'hui. À la manière en effet de ces objets ethniques promis à de nouvelles mondialisations et virtualisations, le personnage volette ici ou là, non sans conserver une part d'opacité qui nous conduit à reprendre la question anthropologique par excellence posée par Montesquieu en son temps : comment peut-on être persan ?' S'il rebondit, c'est aussi dans la fraîcheur d'un bouche-à-oreille activé par les passeurs culturels que sont les conteurs contemporains. Melisdjane Sezer, conteuse et danseuse stambouliote à ses heures, nous servira de guide. Il s'agira d'explorer, ce faisant, des dispositifs d'enquête au plus près du vivant de la parole.

${ }^{1}$ in Lettres Persanes, 30, 1721. 


\section{Herodotos Sezer, curieuse voyageuse, voyageuse curieuse...}

Melisdjane-Melisanda Sezer fait partie de ces voyageurs qui sillonnent les Orients et les ailleurs, toujours à la recherche de contes, de chorégraphies, des mille et une manières possibles de plier les histoires, d'assouplir les corps, d'infléchir les voix. Des journalistes tsiganes l'ont même surnommée Hérodote Sezer. ${ }^{2}$

Ses pérégrinations de fait l'apparentent aux érudits de terrain, qui ont jalonné l'histoire des civilisations et font régulièrement l'objet de commémorations, plus ou moins remarquées du grand public. Ainsi en 2004, les célébrations du tricentenaire de la traduction par Antoine Galland des Mille et une Nuits. Laissons pour l'instant la question de l'orientalisme à Edward Saïd et ses lecteurs, pour retenir ici la figure du premier enquêteur-voyageur qui sut parcourir et se représenter de très vastes espaces. "L'ami des Barbares », le « vieil Hérodote », le " rhapsode en prose ", comme l'appelle l'historien François Hartog ${ }^{3}$, est celui que l'on s'accorde à reconnaître à la fois comme le père de l'histoire et comme le père de la géographie et de l'ethnologie, ou même comme le premier ethnographe du $\mathrm{Maghreb}^{4}$, ou encore, c'est en cela qu'il nous intéresse aussi, comme le père du mensonge. La fameuse Enquête (traduite aussi par Histoires), historiè, menée au v ve siècle avant J.-C., se fait sous le régime tant du voir que de l'ouïr : logoi (récits) et legetai (on dit) martèlent le propos. Ni menteur, ni hâbleur, le voyageur se doit de tout dire, y compris les différentes versions recueillies, ou s'abstient parfois de dire (" je sais, mais je n'écris pas »). La catégorie du croyable, dans ses subtiles gradations, et même le refus de croire servent en fin de compte un faire-croire du récit, lequel pose en définitive la question du vouloir-croire du public, c'est-à-dire la question de la réception et de l'écoute. Est ainsi proposé un nouveau mémorable, entre l'oral et l'écrit, très différent de la matière épique, placée, elle, sous le signe du chant, de l'invocation aux Muses. Les Histoires n'offrent-elles pas à nos yeux et oreilles ébahis une sorte de recueil de contes populaires où le jeu de l'énonciation procède de l'art subtil du conteur? La constante oscillation entre vérité et mensonge est un modèle du genre, tel qu'il se réinvente aujourd'hui, laissant en définitive au destinataire toute liberté de le croire ou pas. Ainsi le miracle d'Arion voguant sur le dos du dauphin est-il, en fin de compte, soumis à enquête par Périandre, l'avisé tyran de Corinthe.

\footnotetext{
${ }^{2}$ Bu da Herodotos Sezer: Et celle-ci [est] Hérodote Sezer, Aksiyon, 23 mai 2005, suite au premier Colloque international organisé en Turquie sur la communauté Rom, 7-9 mai 2005, Edirne.

${ }^{3}$ F. Hartog, Le miroir d'Hérodote, Paris, Gallimard, 2001. Voir aussi Y. Lacoste, La légende de la terre, Paris, Champs Flammarion, 1996.

${ }^{4}$ G. Tillion, Le harem et les cousins, p. 93. Y. Lacoste (op. cit. : 65-66) explique la proche limite des connaissances géographiques d'Hérodote en direction du couchant (Maghreb) par l'obstacle carthaginois; c'est néanmoins lui qui donna le nom d'Atlas aux montagnes de cette contrée.
} 
Comme Hérodote d'Halicarnasse (l'actuelle Bodrum), Melisdjane Sezer, reprenons les mots de F. Hartog", " coud les espaces les uns aux autres ", est "l'agent de liaison qui a souci de lier les espaces " sinon continûment, jusqu'aux limites du monde habité, du moins dans les Orients et divers pays où la poussent ses recherches en matière de conte et de danse. Melisdjane est née dans la même région du monde ${ }^{6}$. Sa ville natale, Mersin, se trouve juste en face de l'île de Chypre-paysage mental ouvert à la mer, à l'extérieur, avant que ne se soit offert à elle le spectacle du Bosphore. Elle se souvient des premières images qui ont marqué son enfance, celles de la magnifique forteresse dite "Forteresse de la fille " (Kizkalesi), construite sur la mer, à quatre-vingts kilomètres au sud de Mersin, l'un de ses lieux habituels de promenade et de baignade. C'est là qu'elle a commencé à se raconter des histoires, rêvant peut-être déjà à la légende attachée au lieu : un devin aurait prédit au roi que sa fille mourrait d'une morsure de serpent; le roi lui a fait bâtir cette forteresse dans la mer, pour la protéger, mais un jour une paysanne apporte un panier de figues ou de raisins, où se trouve un serpent. Du côté de sa mère, turque, il y a l'islam sunnite; mais la famille est venue de Thessalonique en passant par Rhodes et la Crète. Son père, Libanais, catholique maronite, est venu de Beyrouth s'installer au Sud de la Turquie. Du côté paternel, se trouvent des maronites et des orthodoxes. Melisdjane Sezer a toujours connu ce mélange des langues, des cultures, des religions, qui ne participe d'aucune recherche d'originalité, mais imprègne si profondément la vie des gens comme l'œuvre des artistes, tel l'écrivain Amin Maalouf ${ }^{7}$ :

"Je suis né, comme beaucoup de Libanais, avec trois langues dans la bouche : l'arabe, le français, l'anglais. Dans ma jeunesse, la chose paraissait si naturelle qu'il me fallut du temps, et de nombreux voyages, pour comprendre à quel point elle était rare.

Non pas le multilinguisme en soi, que l'on retrouve dans divers pays, sous toutes les latitudes, et qui est d'ailleurs presque toujours un facteur d'enrichissement, ainsi qu'une école de la civilité. Je fais plus précisément allusion à " l'exception levantine » en vertu de laquelle la langue emblématique du monde arabo-musulman cohabite, dans le même esprit, avec des langues occidentales librement choisies, et apprises avec soif. »

Melisdjane Sezer, elle, a quitté Istanbul à dix-neuf ans, après des études au lycée Galatasaray, qui lui ont permis de cultiver son goût de la poésie et de la musique, de se former à la pratique de la danse, aux arts de la scène. Ses premières explorations, au niveau des répertoires de conte et de danse, ont donc pour point de départ la Turquie et, par extension géographique, le Moyen-Orient (pays arabes, Iran, Asie Centrale, Inde) puis les rives de la Méditerranée, avec toujours une prédilection pour les

${ }^{5}$ ibid., p. 505.

${ }^{6} \mathrm{Je}$ m'appuie ici essentiellement sur cinq entretiens enregistrés à Istanbul en septembre 2004, dont la transcription a fait ensuite l'objet de discussions et réécritures (ouvrage à paraître).

${ }^{7}$ A. Maalouf, «Le français a appris la modestie et cela lui a fait du bien », in $L e$ Courrier international. Hors série Culture, mars-avril-mai 2003, p. 44-45. 
îles (Chypre, Corse, Sicile, Zanzibar). Devenue danseuse, chorégraphe, enseignante de danse en 1982 et conteuse en 1987, elle a irréversiblement renoncé, dès la fin de ses études de droit, à la robe d'avocate au profit d'autres formes d'expression. "Femme des allers-retours ", " citoyenne du monde ", comme elle aime à se définir, Melisdjane Sezer s'est forgé une méthode d'enquête qui procède à la fois de la préparation et de l'improvisation, en autodidacte. Depuis presque trente ans, elle cultive donc cette mouvance : aller sur place, sans être forcément en position d'invitée officielle. C'est ainsi que je l'ai accompagnée à Oujda, au Maroc ${ }^{8}$. Dans tous les pays visités (Balkans, Zanzibar, Yémen, Cuba), elle écoute, raconte, rencontre. C'est pour elle une façon de se former, d'enrichir son répertoire et sa personne, au sens moral du terme. Il en sort toujours une création, un spectacle, une prestation partagée au retour. Selon une expression turque que l'on pourrait traduire littéralement par : à la fois de l'école, à la fois de la rue, un processus de rencontres s'opère, qui n'est pas de l'ordre du désorganisé. "Voyageuse curieuse, curieuse voyageuse ", selon ses propres mots, elle ne va pas au hasard, mais s'informe, se documente et part vérifier une hypothèse. Cette démarche prend acte d'une pluralité des mondes possibles, hors des sentiers battus des écoles et autres formations instituées. À sa manière, si proche peut-être de celle du « vieil » Hérodote, Melisdjane Sezer montre en même temps le rôle joué par les autres dans son parcours de conteuse contemporaine, des " autres donneurs de sens ", pour reprendre une expression commentée par Charles Taylor'. Cette exigence d'authenticité peut être rapprochée de l'idéal moral qui se développe dans la conscience moderne, aux yeux du philosophe, et s'élabore non pas dans l'isolement ou l'imitation d'un modèle, mais par le dialogue ${ }^{10}$. Le trait essentiel de la vie humaine, souligne Taylor, est son caractère fondamentalement dialogique : nous apprenons par les échanges avec les autres, d'où l'importance des relations, de la reconnaissance venue de ces éminents "autres », dans le mélange des horizons qui caractérise nos sociétés multiculturelles. Comment trouver sa voie et permettre à chacun d'être au plus près du juste en restant soi-même, tel est aussi le souci constant exprimé en ces termes par la formatrice en matière de conte et de danse qu'est Melisdjane. Cette exigence d'authenticité guide les orientations et les choix artistiques de la conteuse et danseuse, les deux pratiques étant indissociablement liées dans son art et comme sa signature.

\footnotetext{
${ }^{8}$ Mission de recherche dans le cadre d'un projet cnrs (UmR 5647, Maison de l'Orient et de la Méditerranée, Lyon), avril 2001.

${ }^{9}$ Expression que Taylor emprunte à George Herbert Mead (Mind, Self and Society, Chicago, University of Chicago Press, 1934) dans Multiculturalisme. Différence et démocratie, Paris, Champs Flammarion (1992/1994), p. 50.

${ }^{10}$ Ibid., p. 47.
} 


\section{HistolRe d'Une ENQUÊTE ET PREMIÈRE PRÉSENTATION DU PERSONNAGE}

Partie de la Turquie ou pour le dire, avec sa malice, " du nombril de l'Anatolie », Melidsjane non seulement a introduit le personnage en Rhône-Alpes, mais n'a de cesse d'explorer un répertoire longtemps méconnu en France, et auquel sa connaissance de la langue lui donne un accès direct. Ses auditeurs ont bien de la chance, car elle continue inlassablement à récolter un corpus que l'on peut dire " rare ».

Le collectionneur, à l'instar du flâneur, joint l'attitude de l'héritier à une recherche guidée par le hasard. Les fragments arrachés à leur contexte vont produire un nouvel ordre et procèdent d'un penser poétiquement le monde. Ainsi naissent de nouvelles formes et configurations cristallisées qui transforment la mémoire collective à partir des singularités de la rencontre, du voyage. Prenons un exemple qui permettra une première entrée en scène du personnage : Melisdjane et sa découverte, en Ouzbékistan, parmi d'autres statues aux yeux plutôt bridés, d'une statue de Nasreddin accompagné de son âne légendaire :

"C'était à Boukhara, j'allais prendre le car pour aller vers Khiva, qui est une ville magnifique, une sorte de musée à ciel ouvert. En attendant le car, je me promenais et tout d'un coup, je me retrouve dans une cour derrière l'hôtel, du côté où se trouvent les cuisines, et qu'est-ce que je vois là-bas, une sorte de bassin et, au bord, je vois Nasreddin en statue, à taille humaine, avec son âne à taille animale, et, devant l'âne, il y a un livre ouvert : ça, c'est une blague, une histoire de Nasreddin justement, c'est Tamerlan, le boiteux, le méchant, qui dit à Nasreddin : "Puisque tu es censé tout savoir, puisque tu peux tout faire, je t'ordonne ceci, tu vas apprendre à ton âne à lire. " L'histoire commence comme cela, je ne raconte pas toute l'histoire, mais pourquoi cette statue et pourquoi elle se trouve derrière l'hôtel, là où nul client n'est censé aller. Un Ouzbek m'a donné la réponse. Nasreddin dit que son âne est tellement intelligent qu'il peut même apprendre à lire le livre, or le livre dans l'esprit oriental croyant, c'est le Coran. Après la chute de l'urss, avec la montée d'un certain islamisme, des gens se sont offusqués de voir - alors même qu'ils la voyaient jusque-là - cette statue devant l'hôtel. Pour eux l'âne essayant de lire avec un étonnement joyeux constituait une insulte à la religion musulmane. Ainsi ont-ils fait déplacer la statue. Eh bien, c'est encore heureux qu'ils ne l'aient pas cassée! Je le tiens de quelqu'un avec qui j'ai beaucoup sympathisé là-bas et qui m'a raconté l'histoire, l'histoire de l'histoire. La statue est très belle. L'âne est effectivement penché avec les pattes plutôt courbés vers le sol, le regard et le nez tournés vers le livre qui est ouvert. Nasreddin, à côté de lui, arbore un magnifique sourire. "

Nasreddin Hodja est ce fou sage spécifiquement turc ou revendiqué comme tel, le plus souvent accompagné de son âne. Il s'agit ici d'une anecdote piquante, qui en souligne bien la dimension satirique et l'impact politique. Le conte peut se réduire, comme ici, à l'allusion et les nombreuses aventures du bonhomme se traduire par une formule quasi proverbiale, invoquée in medias res, pour commenter une situation. Le 
répertoire pourrait donc fonctionner à demi-mot, il suppose en tout cas une grande complicité, qui relève d'abord d'une compétence culturelle spécifique, à valeur nationale : les blagues, ou facéties, ou Sublimes paroles et idioties de Nasr Eddin Hodja ${ }^{11}$ font partie d'une culture populaire enseignée dans les écoles et colportée dans toutes les langues du tourisme international, à commencer par les principaux pays d'immigration (France, Allemagne, Belgique). Livres, cassettes, BD, maintenant DVD et sites internet : les supports suivent l'actualité de médias de plus en plus interactifs. Les histoires s'enchaînent, et leur brièveté les rend d'autant plus exportables qu'elles reprennent des motifs universels. De plus, le personnage est présent dans tout le bassin méditerranéen et au-delà, notamment au Maghreb, sous le nom de Djoha (Djeh'a, J'ha, Ch'hâ), de Goha en Égypte. En devenant conteuse, Melisdjane a été l'une des toutes premières à débusquer par ricochet un répertoire maghrébin plus ou moins illégitime, enfoui dans les mémoires : par exemple, parmi les historiettes les plus fréquentes, la marmite qui a fait des petits, la maison vendue sauf son clou, la clé ou bague cherchée là où il y a de la lumière, les trois pets de l'âne J'ai maintes fois assisté, dans le cadre de journées de formation interculturelles, à ces irruptions de contage à plusieurs voix, où l'arabe et le turc passaient par le français, à tel point que Melidsjane s'était forgé une formule d'introduction ou de glose ad hoc: Hodja, Djoha, Goha kif kif: sorte de captatio benevolentiae qui a le pouvoir d'immédiatement stimuler une écoute intertextuelle, ouverte à la diversité des cultures, de type plus anthropologique qu'ethnologique, qualité qui pourrait, entre autres, expliquer leur succès actuel.

Ces blagues, car tel est le mot turc qui les désigne (fikra) viennent souvent apporter une note de gaîté, dans des moments de contes, après des contes merveilleux ou des légendes particulièrement émouvantes, qui mettent par exemple en scène des amours contrariés entre nomades et sédentaires, entre chrétiens et musulmans, et se terminent souvent par la mort des héros. Melisdjane puise en effet dans un répertoire venu d'abord de l'Est de la Turquie, un répertoire universel qu'elle n'entend pas édulcorer. Il est bon alors de laisser souffler l'auditoire. Or Nasreddin Hodja fait rire. Du moins la conteuse en est-elle venue à discriminer les histoires qui ne réjouissent que les auditeurs turcs - tant pis, elle sera la seule à en rire - et celles qui se sont diffusées de bouche à oreille ou, plus récemment, par le biais de publications destinées à la jeunesse et de sites Internet. Les traductions en français sont restées longtemps le fait de spécialistes ou de quelques animateurs de centres sociaux (responsables de cours d'alphabétisation ou d'activités culturelles) en ce qui concerne le corpus turc ou maghrébin. Petit à petit, véritable apport des cultures de l'immigration, le personnage est sorti de l'ombre et s'est imposé plutôt sous sa forme turque. Est-ce dû à un simple confort de prononciation ou à la vitalité du personnage? Si ses tribulations passées restent toujours très mystérieuses aux yeux des historiens, le voilà qui

${ }^{11}$ C'est en effet sous ce titre que le lecteur francophone trouvera le recueil le plus fourni (sous-titre « Tout Nasr Eddin, ou presque »), Paris, Éditions Phébus libretto, 2002. 
reprend du service, véritable aubaine pour tous les publics, raison de plus, sans entrer trop dans les détails, pour approcher mieux le personnage et, à travers lui, le phénomène de migration et transformation des imaginaires.

\section{UN GRAND VOYAGEUR, GRAND MÉDIATEUR : UN ART DES PASSERELLES}

Rappelons la complexité et l'opacité vertigineuses des sources. Nasreddin Hodja ou Khodja a peut-être existé, au XIII ${ }^{\mathrm{e}}$ siècle, mais il aurait pu être le bouffon du sanguinaire Timour Lenk, Pied de Fer, Tamerlan le boiteux, le sanguinaire conquérant tatar (fin du XIV siècle) ou aurait pu exister bien avant. Bien des villages turcs revendiquent sa tombe. La ville d'Askhéhir s'enorgueillit de posséder son tombeau et organise chaque année une sorte de pèlerinage avec concours de caricatures. Si les Arabes du Maghreb ne le connaissent pas sous ce nom, explique René Basset ${ }^{12}$, ils possédaient plusieurs types originaux dont la sottise était proverbiale. Chez les Arabes d'Égypte, il est le Kodja Nasr-eddin Djoha er Roumi. A-t-il essaimé au Maghreb, à la suite de la conquête turque, où il aurait pris le nom de Djoh'a, ou inversement estce par la voie de la traduction de recueils arabes en turc que le personnage de Djoh'a est passé en Turquie, où il s'est trouvé identifié avec un certain Nasr eddin Hodja dont l'existence est pour le moins douteuse? À l'inverse, les traductions maintes fois remaniées de recueils turcs en arabe ont procuré, au XVII ${ }^{\mathrm{e}}$ siècle, d'autres sources de diffusion. Galland a vraisemblablement puisé dans une recension arabe dérivée du turc plusieurs anecdotes qu'il a traduites dans ses Paroles remarquables des Orientaux, addition à de la Bibliothèque Orientale de d'Herbelot. C'est sous le nom de Djoh'a, semble-t-il, que le personnage a pu passer en Italie par la Sicile (Giufà), et même gagner l'Albanie (Guicha), les Berbères le transformant en Si Djoh'a ou Si Djeh'a, pour un usage modéré, au profit de nigauds locaux. D'autres sources le présentent comme le contemporain et proche voisin du poète et grand mystique persan Rûmi (1207-1273), qui passa sa vie à Konya en Anatolie. Le nom de ce dernier est Mohammad, son nom honorifique est Jâlâl ud-Dîn (Splendeur, Majesté de la Religion); parmi les titres qui lui sont le plus souvent attribués de son vivant (Mowlânâ : notre Seigneur, ou Khodâvandegâr : notre Maître), l'on retrouve des noms attachés ici ou là à notre personnage ${ }^{13}$. Les soufis ont colporté maintes histoires du divin Hodja, "l'idiot accompli ", comme le rappelle Jean-Louis Manaury, dont les sottises donnent à voir l'absurdité du monde et doivent élever les disciples à une connaissance

\footnotetext{
${ }^{12} \mathrm{R}$. Basset, «Recherches sur Si Djoh'a et les anecdotes qui lui sont attribuées » in J. Dejeux, Les fourberies de Si Djeh'a. Contes kabyles, Paris, La Boîte à Documents, 1987. Pour un aperçu du personnage dans la littérature arabe, se reporter à J.-J. Schmidt, Le livre de l'humour arabe, Arles, Actes Sud Sindbad, 2005.
}

${ }^{13}$ Se reporter pour plus de détails à la présentation de Manijeh Ortega, Le sens de l'amour chez Rûmi, livre bilingue avec cassette, Dervy, 2004. 
plus haute. Ainsi prennent sens ces histoires obscures qui semblent procéder par antiphrases, comme celle de la chemise prise pour le voleur : Hodja croit avoir aperçu un voleur la nuit dans son jardin, il va voir et envoie une pierre sur la forme blanche, qui n'est autre que sa chemise en train de sécher et, rapportant l'affaire à sa femme, s'exclame : "Quelle chance que je ne me sois pas trouvé dedans! ». Entre les recueils de plaisanteries et la transmission orale proprement dite, l'enchevêtrement des circulations brouillent les pistes et quelques repères ne sont pas inutiles pour apprécier la saveur parfois déconcertante du personnage pour le non-initié.

Les Turcs en tout cas ne nomment jamais ce personnage que Nasreddin Hodja. En turc (orthographié hoca), Hodja désigne le savant, celui qui sait, qui possède la connaissance, le savoir religieux. Même dans un milieu laïque, le mot hodja signifie «celui qui enseigne » et peut désigner un professeur. Il est parfois appelé effendi, autre titre de respect que l'on pourrait traduire par " distingué ", " comme il faut ». Parfois il semble âgé, d'autres fois il paraît jeune ou dans la force de l'âge, il est ordinairement marié et surtout quasi inséparable de son âne. Serait-il aujourd'hui chômeur, comme le suggère Jean Dejeux? D'une façon générale, il est parmi les plus démunis, et use de roublardise et de stratagèmes peu recommandables. En tout cas, il en remontre aux puissants : les savants, les dévots, ceux qui s'entourent d'une honorabilité publique. Il s'arrange pour trouver en lui-même les ressources nécessaires à sa défense et, dans l'adversité, n'a d'autres armes que son astuce. Il peut se fâcher, il peut avoir beaucoup d'humour, il aime sa personne et jouit de la vie, son érotisme se prêtant même à des anecdotes hautement scatologiques. Humour et sagesse sont de fait indissociables du personnage. Dans certains endroits, on le traite de fou, dans d'autres endroits, il n'y a pas plus savant que lui, il allie la naïveté à l'intelligence et cette feinte naïveté le rend intouchable. Tamerlan lui-même ne peut lui faire de mal, comme dans cette histoire, parmi d'autres qu'aime bien raconter Melisdjane, où l'empereur l'invite à sa table, sous la tente, pour essayer de faire rire à ses dépens et se trouve finalement le dindon de la farce. Du melon est servi à tous les invités, mais les domestiques ont reçu l'ordre de récupérer les écorces et de les accumuler en douce devant l'assiette de Nasreddin. L'empereur alors fait remarquer qu'il doit être bien pauvre et affamé pour s'être tant empiffré de ce mets délicat. Mais il trouve vite la riposte : bien plus affamés sont tes invités, qui ont même mangé l'écorce.

Les savants, dans leur vanité dédaigneuse, ne s'en sortent pas mieux, en croyant le piéger avec des questions impossibles : «On a entendu tellement dire que tu es un homme très sage, avec beaucoup de connaissances, on voudrait te poser quelques questions pour voir un petit peu. » Et de demander : "Combien d'étoiles y a-t-il dans le ciel? » La réponse fuse : "Autant que de poils sur le dos de mon âne. » Et quand l'un d'eux lui dit : "Mais enfin, comment peux-tu être aussi sûr? ", il répond : " Si tu ne me crois pas, tu n'as qu'à compter. » Peu importe l'embarras créé, la réponse est toujours d'une subtilité éblouissante. Nasreddin incarne la liberté de parole, digne pendant masculin de Schéhérazade. 
Ce que nous pouvons retenir de ce bref panoramique, c'est que Nasreddin Hodja aujourd'hui opère des connivences et joue un rôle de médiateur culturel. Plus qu'il ne se fond dans les populations locales, il en atteste les bigarrures méditerranéennes et opère des métissages. Il est devenu quasi omniprésent dans les cercles de conteurs et appartient à tout le monde. Les élèves des écoles primaires et de collège, dès les premières séances de formation au conte, se l'approprient avec un bonheur manifeste et sont les premiers à vouloir le raconter. Juifs, musulmans, chrétiens franchissent l'obstacle des religions et se retrouvent dans le rire universel qui sanctionne la bêtise, la vanité, l'arrogance, la lâcheté et toutes les formes d'abus de pouvoir ${ }^{14}$. La barbe, l'embonpoint et le turban du bonhomme permettent toutes les caricatures. Nasreddin et son âne courent sur toutes les bouches, sans mettre quiconque en danger : sa statue à Boukhara ne fut même pas cassée, ni remisée, mais juste déplacée et elle alimente encore nos imaginaires.

\section{Conte et RÉCIt DE CONTE}

L'art du conte touche ici à l'excellence, puisque des connivences se sont construites, en situation interculturelle, qui permettent le plein exercice de ce que Paulette Galand-Pernet définit comme un plaisir à la fois esthétique et herméneutique, c'est-à-dire lié au réseau des significations $^{15}$. Nous retrouvons la dimension, fût-ce à toute petite échelle, de la culture de masse que fut le conte populaire, unissant encore à la fin du XVIIe siècle et au XVIIIe les lettrés et gens de cour et le monde des paysans et des nourrices. La variation du conteur peut s'apprécier pleinement sur fond d'intervocalité, pour reprendre le mot de Paul Zumthor : s'ajoute au plaisir du texte le plaisir partagé de l'œuvre performée. Melidsjane, par sa connaissance de la langue turque et des civilisations turquesottomanes, par ses voyages lointains sur les traces de Nasreddin Hodja, peut encore prétendre au plaisir de la nouveauté, de la rareté, qui serait une des modalités de la variation, mais ce plaisir ne cesse de s'amenuiser, vu l'accessibilité croissante des sources, Internet aidant. Elle ne joue pas moins efficacement avec les petites différences qui font apprécier le retour de la même histoire, dans une brièveté qui rend d'autant plus sensible le moindre écart. Ce plaisir lié à la mouvance des textes et des voix, je lui ai attaché, au fil de mes travaux, le nom de variance, qui rime aussi avec itinérance, autre mot cher à Zumthor. Melidsjane Sezer, conjuguant ses propres déplacements avec ceux de Nasreddin, nous en donne une pleine expérimentation sur un régime qui mêle le conte et le récit de conte, invente une forme orale de carnet de voyage érigé en œuvre d'art. Le voyage est d'autant plus merveilleux qu'il s'est effectivement passé et s'apparente aux formes anciennes et volontiers mythiques du

${ }^{14}$ A. Nahum, Humour et sagesse judéo-arabes. Ch'hâ, proverbes et contes, Paris, Desclée de Brouwer, 1998.

${ }^{15}$ P. Galand-Pernet, Littératures berbères. Des lettres. Des voix, Paris, Puf, 1998. 
récit de voyage. Conteur-viator, tel serait l'un des avatars du conteur de tradition contemporaine. Or le voyageur continue à fasciner le sédentaire, même si ce dernier partage de plus en plus avec lui l'expérience du dépaysement, et peut-être d'autant plus. À la manière de Cercamon, l'un des plus anciens troubadours ainsi très justement surnommé « Parcourt-le-monde $\star^{16}$, Melisjdane Sezer, telle une magicienne, réintroduit la dimension du temps, le temps du voyage qui est temps de la recherche, de l'itinérance en des lieux où les gens ne se risquent guère et plutôt en groupe, alors même que nos nouveaux modes de communication tendent à abolir le temps, à neutraliser l'inconnu et même l'aléatoire. Ainsi l'écoute du conte en est-elle renouvelée. En s'impliquant comme voyageuse, conteuse, chercheuse, elle sait ménager la surprise du même et de l'autre, ce qui est une façon d'initier ses divers publics à l'art de la parole comme mouvement, comme danse. Nasreddin Hodja en fournit ici un exemple, où apparaît l'importance du livre dans la capture de l'oralité. L'histoire se passe à Zanzibar, l'île où se tissent depuis tant de siècles les cultures africaines, orientales, indiennes. Partie à la recherche de rythmes, Melisdjane en est revenue non seulement avec la création, inspirée d'une légende, Parce que Zanzibar, mais aussi avec un tout petit ouvrage, The Jokes from Zanzibar, où elle a fait cette trouvaille, dont elle nous donne la traduction :

C'est un homme qui a prêté de l'argent à un voisin, qui tarde à le lui rendre, alors cet homme va vers la maison de son voisin pour réclamer l'argent. Il a entendu, il a même aperçu le voisin, qui est à l'étage. Par sa fenêtre, le voisin lui aussi le voit arriver et dit à son fils : " Dis à cet homme que je ne suis pas là. » Et il s'éclipse. L'autre arrive au bas de la maison. Sûr de trouver son voisin, il frappe et voit le fils qui sort sa tête par la fenêtre : «Eh, qu'est-ce que tu veux? - Je voudrais voir ton père! - Ah, mais mon père n'est pas là, il avait des affaires à faire. - Ah bon, il est parti! D'accord, la prochaine fois, tu diras à ton père qu'il n'oublie pas sa tête à la fenêtre, quand il part. »

Voilà indéniablement une blague dont Nasreddin Hodja pourrait être le héros. Il se peut que les contacts de cultures expliquent certains phénomènes de passerelle, alimentent telle hypothèse propre à satisfaire une curiosité historique. Il est tout aussi divertissant de s'en tenir au simple plaisir de la surprise et d'observer tout simplement que, sous toutes les latitudes, apparaît ce personnage (anonyme ou identifié) porteur des différentes facettes de l'être humain, que ce soit en Inde, en Bulgarie (où le rusé Hitar Pétar dame le pion à Nasreddin pour des raisons politiques, ce dernier y faisant figure d'envahisseur culturel), ou encore en Languedoc et Roussillon ou dans quelque autre endroit insoupçonné ${ }^{17}$. S'il n'y a plus d'espace terrestre à découvrir, d'autant plus précieuses

${ }^{16}$ P. Zumthor, La mesure du monde, Paris, Seuil, 1993, p. 168.

${ }^{17}$ Voir les différents volumes Sagesses et malices de Nasreddin, le fou qui était sage (Paris, Albin Michel, 2000), Sagesses et malices de la Perse (2001), Sagesses et malices de Birbal, le Radjah (2002), Sagesses et malices de Pierre le rusé, dit Hitar Pétar (2005); voir aussi Claude Seignolle : Contes, récits et légendes des pays de France, vol. 3, Paris, Omnibus, 1997. 
sont les cartographies mouvantes de l'imaginaire. La conteuse, qui se dit parfois la petite-fille de Nasreddin Hodja ou l'une de ses petites-filles, ne reniera en aucune manière les nombreux frères ou cousins germains de son aïeul, tel Birbal venu de l'Inde. Entre l'oral et l'écrit, les mélanges sont inépuisables et féconds, avec toujours cette évidence tremblante, selon les mots de Melisjdane, de découvrir sous la nouveauté un air connu.

Nasreddin Hodja nous invite donc encore et toujours à de subtils jeux de miroirs. Le bouche-à-oreille passe aujourd'hui par le numérique, qui apporte encore un faible relais à la vive voix et ne saurait remplacer la plénitude de la présence, mais active les rencontres et les éclats de rire. En tout cas, autour du conte et du récit de conte, de nouvelles modalités de collaboration s'inventent entre conteur et chercheur, selon des nomadismes en partage. C'est du moins en ce sens que s'orientent mes recherches. 